\title{
Predictive factors for achievement of mucosal healing by budesonide 2-mg foam in ulcerative colitis: a pooled analysis of data from two clinical trials
}

\author{
Toshifumi Hibi ${ }^{1}$, Makoto Naganuma ${ }^{2}$, Eisei Oda ${ }^{3}$, Yoji Yamada ${ }^{4}$, Yoshitomo Chujoh ${ }^{5}$, Ryoichi Yoshihara ${ }^{6}$, \\ Mamoru Watanabe ${ }^{7}$ \\ ${ }^{1}$ Center for Advanced IBD Research and Treatment, Kitasato University Kitasato Institute Hospital, Tokyo; ${ }^{2}$ Division of Gastroenterology and \\ Hepatology, Department of Internal Medicine, Keio University School of Medicine, Tokyo; ${ }^{3}$ Medical Statistics Division, AC Medical Inc., Tokyo; \\ ${ }^{4}$ Clinical Development Department and ${ }^{5}$ Medical Department, EA Pharma Co., Ltd., Tokyo; ${ }^{6}$ Medical Research, Kissei Pharmaceutical Co., \\ Ltd., Tokyo; ${ }^{7}$ Department of Gastroenterology and Hepatology, and TMDU Advanced Research Institute, Tokyo Medical and Dental University, \\ Tokyo, Japan
}

Background/Aims: Mucosal healing (MH) of distal lesions in ulcerative colitis (UC) has recently been confirmed with budesonide 2-mg foam (BF) treatment in 2 clinical trials; however, few studies have investigated the predictive factors for complete MH. Methods: We conducted a post hoc analysis using pooled data from phase II and III clinical trials evaluating the efficacy and safety of BF for UC. Additionally, we analyzed the relationships between complete MH and baseline factors and clinical symptoms from baseline to week 6. Results: Among the 291 Japanese patients from the 2 pooled clinical studies, 119 patients in the BF twice a day group and 117 in the placebo group were included in the full analysis set. The proportion of patients with a rectal bleeding (RB) subscore of 0 was significantly higher in the BF group than in the placebo group after a 5-day treatment $(P<0.05)$. After a 2-day treatment, significantly more patients in the BF group had a stool frequency (SF) subscore of 0 than patients in the placebo group $(P<0.05)$. Multivariate analysis showed that complete MH at week 6 was influenced by baseline SF subscore and 5-aminosalicylic acid (5-ASA) enema or suppository use ( $P=0.0086$ and $P=0.0015$, respectively). The relationship between complete MH at week 6 and RB subscore after week 2 was also confirmed. Conclusions: Normal SF at baseline, history of 5-ASA topical product use, and elimination of RB after week 2 are suggested predictors of complete MH at week 6 with twice-daily BF treatment. (Intest Res 2020;18:56-68)

Key Words: Colitis, ulcerative; Budesonide foam; Complete mucosal healing

\section{INTRODUCTION}

Ulcerative colitis (UC) is an idiopathic, nonspecific inflammatory disease that affects the colonic mucosa. The disease is chronic, with repeated episodes of relapse and remission, and the main symptoms are rectal bleeding (RB), diarrhea, and

Received June 10, 2019. Revised November 15, 2019.

Accepted November 15, 2019.

Correspondence to Toshifumi Hibi, Center for Advanced IBD Research and

Treatment, Kitasato University Kitasato Institute Hospital, 5-9-1 Shirokane,

Minato-ku, Tokyo 108-8642, Japan. Tel: +81-3-5791-6487, Fax: +81-3-

5791-6489, E-mail: thibi@insti.kitasato-u.ac.jp abdominal pain. UC is characterized by lesions that spread continuously from the rectum, progressing towards the ascending colon, which can extend throughout the entire colon. Depending on its extension, UC is classified as proctitis, leftsided colitis, or pancolitis. Topical therapy, consisting of enemas or suppositories, is used to control the distal lesions and is usually the first choice of clinicians based on European and American clinical practice guidelines. ${ }^{1,2}$

Budesonide 2-mg foam (BF) in a single injection can be distributed from the rectum to the sigmoid colon. ${ }^{3}$ Although BF is a glucocorticoid with high receptor affinity ${ }^{4}$ and has potent 
anti-inflammatory action at the treatment site, it is quickly metabolized in the liver with low systemic exposure. ${ }^{5}$ Foam preparations are expected to reduce leakage, a problem with liquid enemas, ${ }^{6}$ because foam has high retention within the rectum. Additionally, with this treatment method, BF can achieve a wider range of distribution than a suppository. ${ }^{3}$ Currently, BF is widely prescribed in Japan for UC; for non-severe cases, the recommended dosage is usually a single rectal administration of $\mathrm{BF}$ (containing $2 \mathrm{mg}$ ) given twice daily. It is recommended that patients are carefully monitored during BF use, and the necessity of continuing BF therapy for 6 weeks should be carefully considered.

Mucosal healing (MH) has been regarded as the therapeutic goal of UC treatment. Complete MH has been associated with reduced subsequent rates of relapse, ${ }^{7,8}$ hospitalization, ${ }^{9}$ and surgery. ${ }^{10}$ Additionally, patients with a Mayo endoscopic subscore (MES) of 0, indicating complete $\mathrm{MH}$, have a better prognosis than those with an MES of $1 .{ }^{11}$ We recently conducted randomized clinical trials targeting an MES of 0 as an efficacy endpoint of BF treatment.

The superiority of BF twice a day for 6 weeks over placebo for attaining complete MH was confirmed in UC patients with active mucosal inflammation in phase II and phase III clinical trials in Japan; ${ }^{12,13}$ however, few studies have investigated the predictive factors for $\mathrm{MH}$ in patients treated with BF. Furthermore, there is a concern that because topical therapy administration is more complicated than that of oral medication, patient adherence to topical therapy is poor. ${ }^{14}$ Although patients typically show improvement in RB symptoms early in treatment, Naganuma et al. ${ }^{13}$ found that patients may require 6 weeks of twice-daily treatment to achieve complete MH. For the purpose of improving patient adherence to $\mathrm{BF}$ treatment twice a day for 6 weeks, we conducted a post hoc analysis of the pooled data from 2 clinical trials on BF conducted in Japan to explore the demographic and clinical factors that affect prognosis and to determine the predictors of the therapeutic effect of BF.

\section{METHODS}

\section{Ethical Considerations}

The phase II and III clinical trials ${ }^{12,13}$ from which the data were obtained were conducted in compliance with the Declaration of Helsinki and Good Clinical Practice. The institutional review board of each center approved the protocol. All patients provided written informed consent.

\section{Participants and Treatment Intervention}

We conducted the present analysis using pooled data from phase II and phase III clinical trials (Japic CTI-132294 and Japic CTI-142704) evaluating the efficacy and safety of BF (2 $\mathrm{mg} / 25 \mathrm{~mL}$ ) in patients with UC in Japan. The details of the study designs, inclusion criteria, interventions, randomization, and blinding have been reported previously. ${ }^{12,13}$ Briefly, patients were randomized at a ratio of 1:1:1 into 3 groups in the phase II clinical trial as follows: BF (once/day), BF (twice/day), or placebo foam. In the phase III clinical trial, patients were randomized at a ratio of 1:1 into 2 groups as follows: $\mathrm{BF}$ (twice/ day) or placebo foam.

\section{Analysis Procedures}

A Modified Mayo Disease Activity Index (MMDAI) score was used to assess disease activity. The enrollment criteria were a stool frequency (SF) subscore of $0-2$, RB subscore of 1-2, endoscopic subscores of 2 in the segment from the rectum to the sigmoid colon and 0-1 in the adoral segment beyond the sigmoid colon, and $\geq 12$ weeks since UC diagnosis.

BF was administered for 6 weeks. Concomitant therapy with oral 5-aminosalicylic acid (5-ASA) agents, oral salazosulfapyridine agents, or probiotics in stable doses was permitted. The use of the following drugs and therapies was prohibited: 5-ASA rectal preparations or suppositories, salazosulfapyridine suppositories, corticosteroid preparations, cytapheresis, immunomodulators, antitumor necrosis factor antibody preparations, and surgical treatment for UC.

Because the approved BF regimen was twice-daily administration, patients administered BF once a day in the phase II study were excluded from the pooled population in this analysis.

\section{Definition of Endpoints and Parameters}

The efficacy endpoints were complete MH, clinical remission (CR), elimination of RB, and normalization of SF. CR was defined as an RB subscore of 0 , endoscopic subscore of 0 or 1 , and either an SF subscore of 0 or a decrease by at least 1 from baseline using the MMDAI subscore ${ }^{15}$ Complete MH was defined as an MES of 0 . The present active phase for each patient was defined as the period between the start of remission induction therapy in this active phase and study enrollment completion. Patients with a first attack were defined as any patient diagnosed with UC who were enrolled in the study during the first active phase of UC. A relapsing/remitting clinical course was defined as patients who had experienced CR of UC in the past and were enrolled in the study during a flare-up phase. 
These definitions were the same as those used in the 2 aforementioned clinical studies. ${ }^{12,13}$

\section{Outcomes}

We evaluated the efficacy and safety of the approved dose of BF by using pooled data from the phase II and phase III clinical trials evaluating the efficacy and safety of BF for 6 weeks in UC patients.

The relationships between $\mathrm{CR} / \mathrm{complete} \mathrm{MH}$ at week 6 and the clinical characteristics of patients were analyzed. Additionally, we analyzed the relationships between $\mathrm{CR} /$ complete $\mathrm{MH}$ at week 6 and RB and SF subscores from baseline to week 6 .

\section{Statistical Analysis}

Baseline demographics and clinical characteristics were summarized and compared between treatment groups using appropriate descriptive statistics and statistical tests. The number of patients who achieved the defined efficacy endpoints (complete $\mathrm{MH}, \mathrm{CR}$, and elimination of $\mathrm{RB}$ ) and the achievement rates at week 6 were calculated, along with the $95 \%$ CI, by treatment group. The difference in achievement rates among the treatment groups was evaluated using the Fisher exact test, and a $P$-value was calculated.

The number of patients who achieved the defined efficacy endpoints, the number of patients who did not achieve the defined efficacy endpoints, and each of the achievement rates were calculated and classified by patient characteristics. Additionally, the proportion of patients who achieved each of the efficacy endpoints was evaluated using comparative tests, and each $P$-value was calculated. Statistical analyses were conducted at a significance level of 0.05 (two-sided).

To evaluate the factors affecting the achievement of efficacy endpoints, the number of patients who achieved each of the efficacy endpoints was counted and each achievement rate was calculated and classified by patient characteristics. Additionally, a logistic regression analysis was conducted with the efficacy endpoint as the dependent variable and patient background characteristics as the explanatory variable-age, sex, body weight, smoking habits, disease duration, first attack/relapse/remitting classification, duration of present active phase, extent of past lesions, MMDAI score, subscore for endoscopic, $\mathrm{RB}$ and SF, physician's general evaluation score, previous medication for UC (i.e., oral 5-ASA preparation, 5-ASA enema/suppository, adrenocortical hormone, cytapheresis, immunomodulator, anti-TNF $\alpha$ antibody, other [excluding other investigational drugs], unknown or other investigational drugs), con- comitant drugs such as oral 5-ASA formulation, and severity: total of each subscore (endoscopic, RB, and SF); an odds ratio was calculated along with the $95 \% \mathrm{CI}$ and $P$-value. The results of the analyses are presented using the following 3 models: univariate (the explanatory variable is one item), multivariate first (the explanatory variables are all items with $P<0.10$ in the univariate model), and multivariate final (the explanatory variables with $P<0.05$ are selected using the step-down procedure). Additionally, an exact logistic regression was used to estimate regression parameters if the model was inappropriate when the data structure was near separation.

The number of patients who achieved complete MH was determined, and the achievement rate at week 6 was calculated using RB or SF subscores. The number of patients who achieved CR was determined, and the achievement rate at week 6 was calculated using RB or SF subscores. In addition, the Cochran-Armitage test was used to evaluate the relationship between complete MH or CR at week 6 and the RB or SF sub-

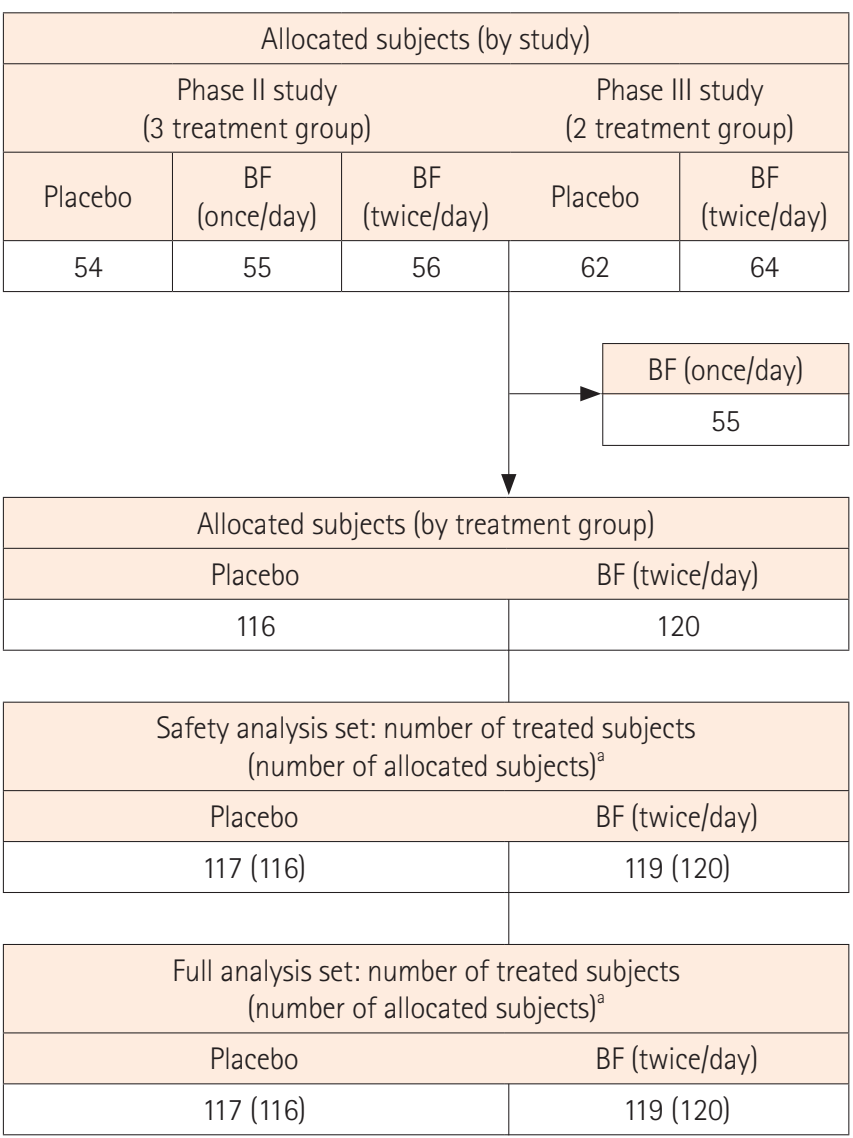

Fig. 1. Disposition of the patients. ${ }^{a}$ Number of allocated patients does not coincide with that of treated patients because one patient allocated to BF was actually treated with placebo. BF, budesonide 2-mg foam. 
score for 6 weeks, and each $P$-value was calculated. Furthermore, proportional curves for patients with an RB or SF subscore of 0 after BF treatment were calculated with the last observation carried forward method to impute the data. The data
(RB subscore, endoscopic subscore, and SF subscore) for patients who discontinued the study at less than 6 weeks of treatment were also imputed with the last observation carried forward method.

Table 1. Baseline Demographics and Clinical Characteristics

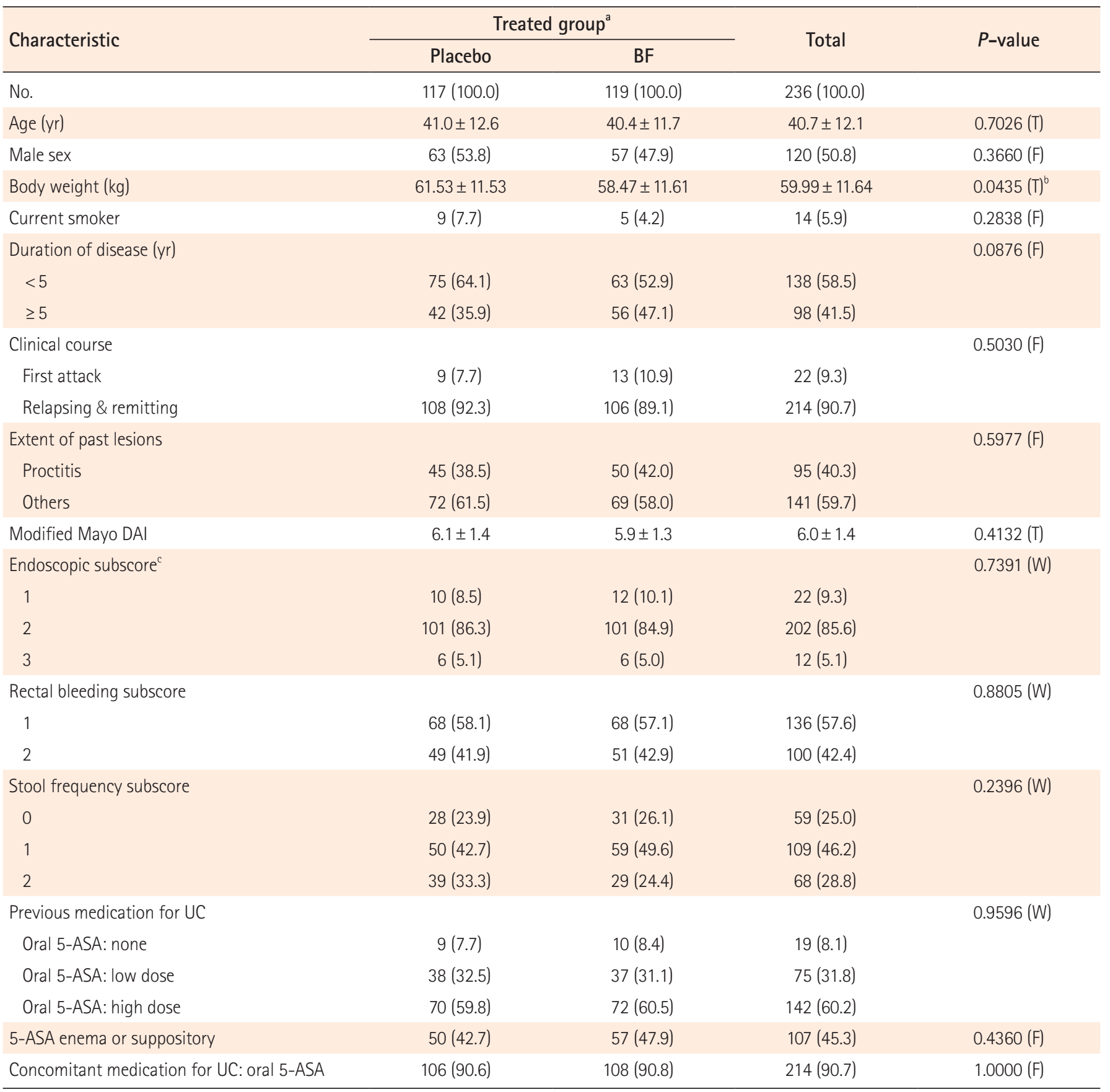

Values are presented as number (\%) or mean \pm SD.

${ }^{2}$ Twice/day.

${ }^{b} P<0.05$.

${ }^{C}$ Assessed by the investigator in the phase II study ${ }^{12}$ and by the central committee in the phase III study. ${ }^{13}$

BF, budesonide 2-mg foam; DAl, disease activity index; 5-ASA, 5-aminosalicylic acid; T, t-test; F, Fisher exact test; W, Wilcoxon test. 


\section{RESULTS}

\section{Disposition of Patients}

Fig. 1 shows the patient disposition. Among the 291 patients from the 2 pooled clinical studies, 55 who received BF once a day in the phase II study were excluded. The remaining 236 patients, comprising 119 in the BF twice a day group (B) and 117 in the placebo group $(\mathrm{P})$, were included in the full analysis set. Because 1 patient who was allocated to BF was actually treated with placebo, the number of allocated patients does not coincide with that of treated patients.

\section{Patient Background and Results of the Efficacy Endpoint Evaluation}

Table 1 shows the baseline demographics and clinical characteristics of patients. Patients in groups $B$ and $P$ had mean $( \pm$ SD) ages of $40.4 \pm 11.7$ and $41.0 \pm 12.6$ years, and $47.9 \%$ and $53.8 \%$

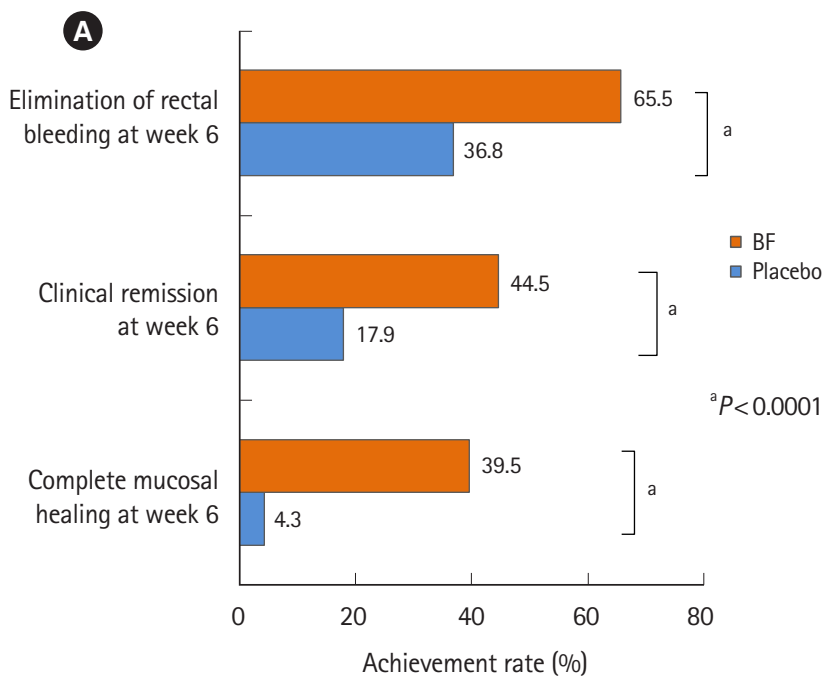

C

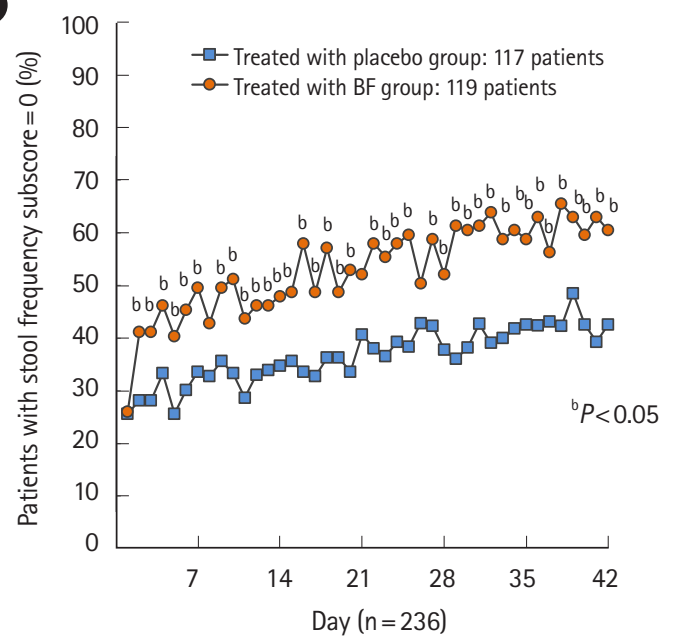

were male, respectively. Regarding past lesions, $42.0 \%$ of patients in group B and $38.5 \%$ of patients in group $\mathrm{P}$ had proctitis. The MMDAI scores were $5.9 \pm 1.3$ in group B and $6.1 \pm 1.4$ in group P. In group B, $84.9 \%$ of patients had an endoscopic subscore of 2, compared with $86.3 \%$ in group P. Except for body weight, there were no substantial differences in the baseline demographic or clinical characteristics between the 2 groups.

Fig. 2A shows the clinical efficacy endpoints. The achievement rates $(95 \% \mathrm{CI})$ for elimination of $\mathrm{RB}$ in groups $\mathrm{B}$ and $\mathrm{P}$ were $65.5 \%$ (56.6\%-73.5\%) and 36.8\% (28.6\%-45.8\%), respectively $(P<0.0001)$. The achievement rates for complete $\mathrm{MH}$ in groups B and P were 39.5\% (31.2\%-48.5\%) and 4.3\% (1.8\%9.6\%), respectively $(P<0.0001)$. The achievement rates for $\mathrm{CR}$ in groups B and $\mathrm{P}$ were $44.5 \%$ (35.9\%-53.5\%) and 17.9\% (12.0\%$25.9 \%)$, respectively $(P<0.0001)$. The results for all efficacy endpoints showed that patients in group $\mathrm{B}$, who received the authorized BF dosage, had significantly higher efficacy rates than

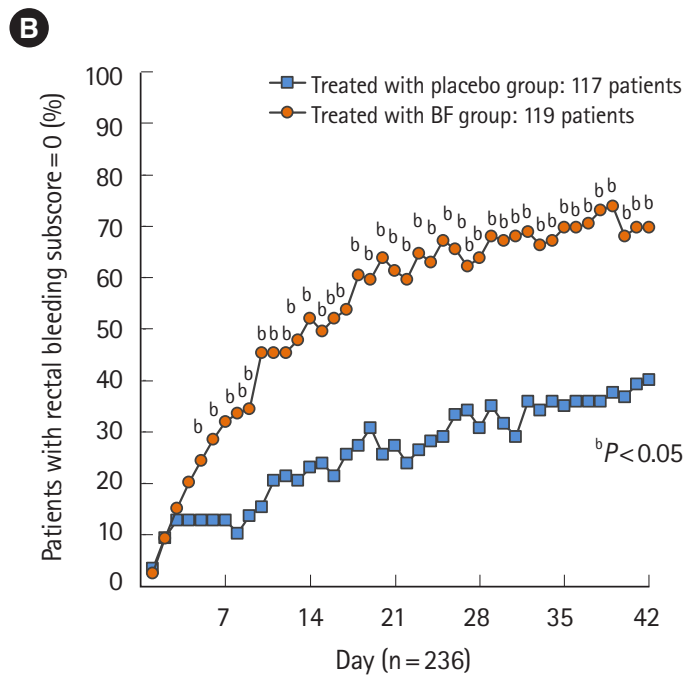

Fig. 2. Results of integrated analysis of data from BF (twice/day) group and placebo group in the phase II and phase III clinical trials. (A) Achievement rate for elimination of rectal bleeding, clinical remission and complete mucosal healing at week 6. (B) Transition chart of the percentage of patients with a rectal bleeding subscore of 0 during 6 weeks of administration. (C) Transition chart of the percentage of patients with a stool frequency subscore of 0 during 6 weeks of administration. BF, budesonide 2-mg foam. 


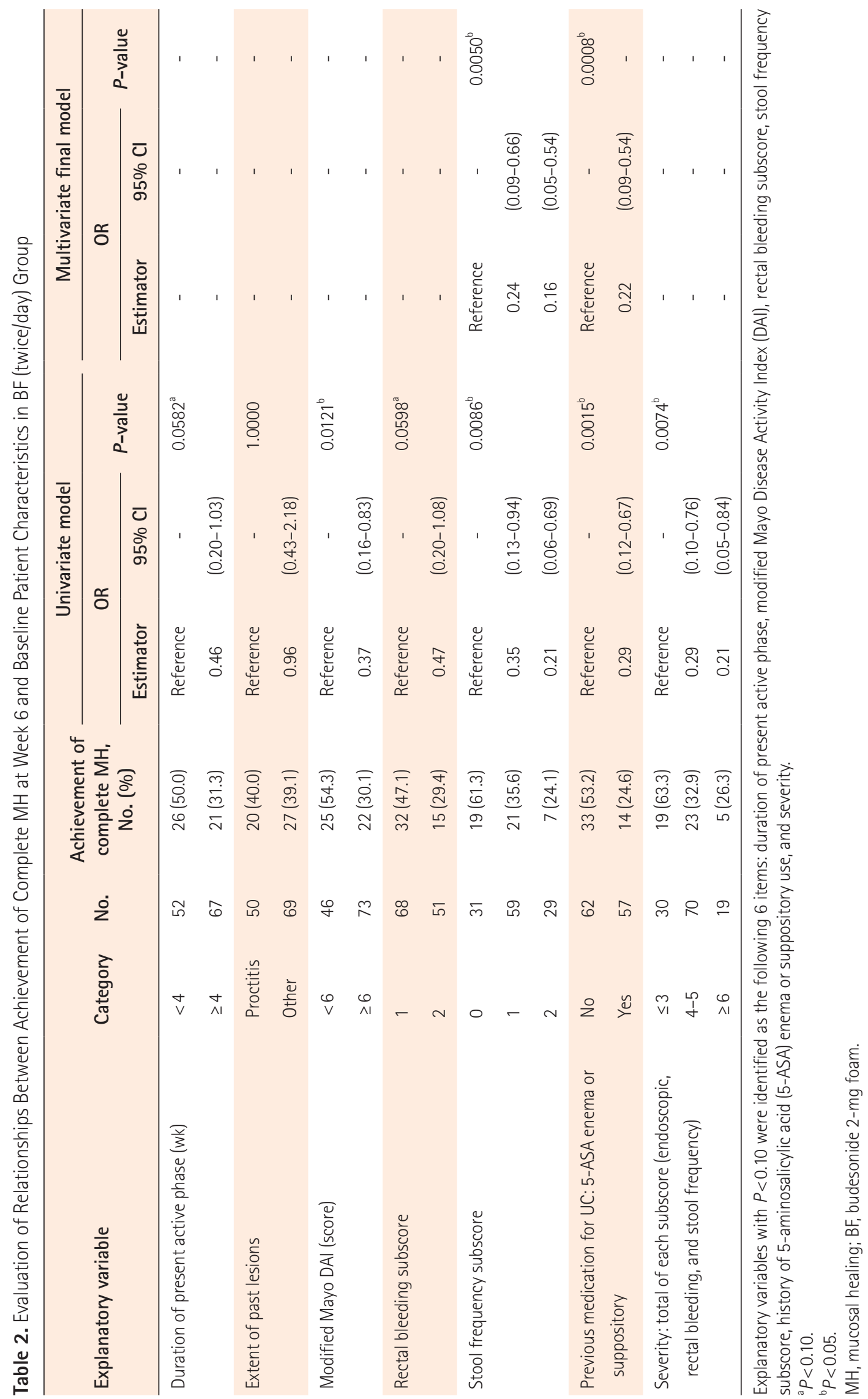




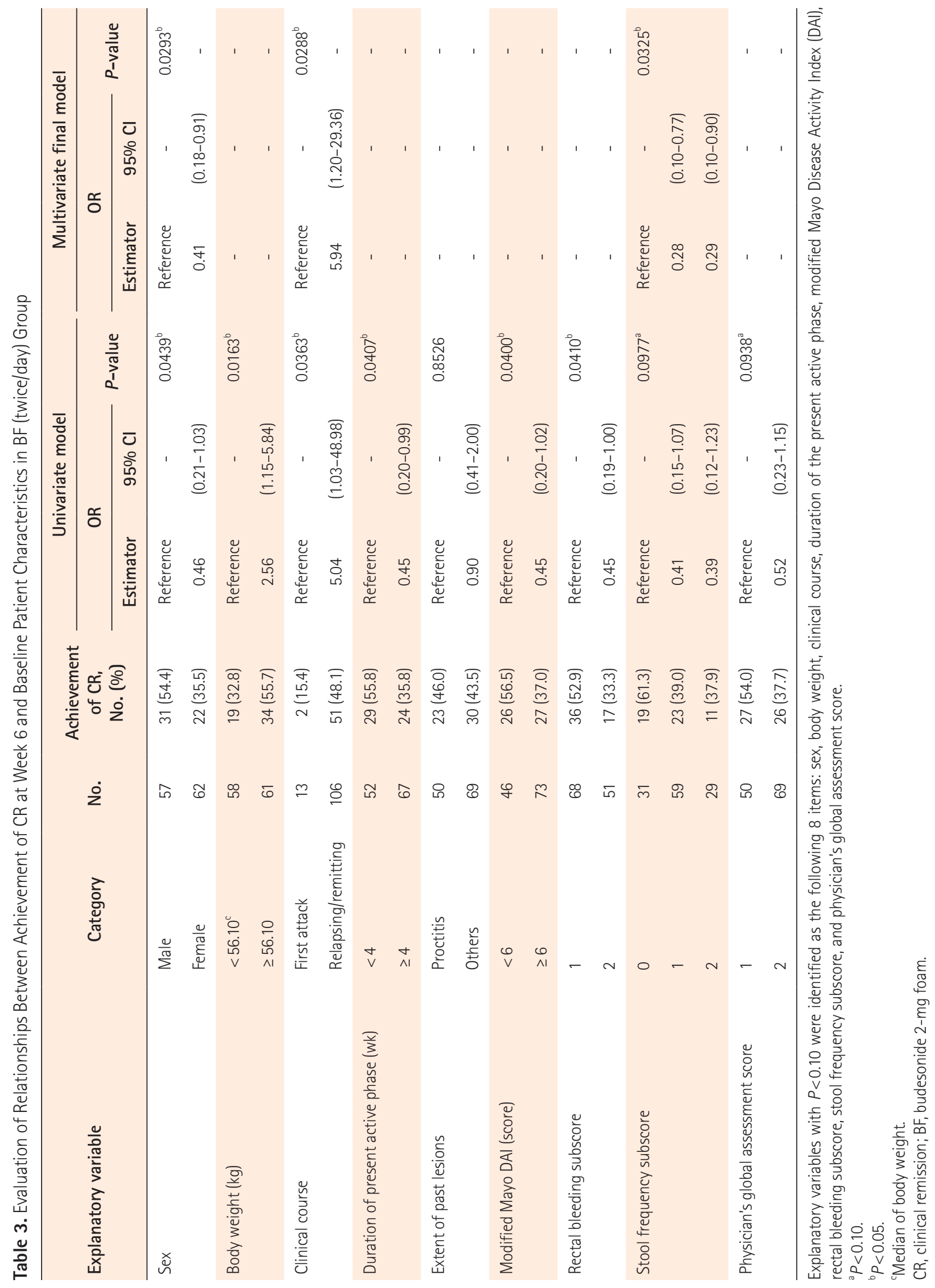


group P.

The proportion of patients with an RB subscore of 0 was significantly higher in group $B$ than in group $P$ after 5 days of treatment $(P<0.05)$ (Fig. 2B). In addition, the proportion of patients with an SF subscore of 0 was significantly higher in group $B$ than in group $P$ after 2 days of treatment $(P<0.05)$ (Fig. 2C).

\section{Evaluation of the Relationships between Complete MH or CR at Week 6 and Patient Characteristics at Baseline}

Table 2 shows the logistic regression analysis for complete $\mathrm{MH}$ in the univariate and multivariate analyses. In the univariate analyses, we found that the following 6 variables influenced complete MH: duration of the present active phase, MMDAI, baseline RB subscore, baseline SF subscore, history of 5-ASA enema or suppository use, and severity. Multivariate analysis revealed that baseline SF subscore and history of 5-ASA ene$\mathrm{ma}$ /suppository use were independently associated with complete MH at week 6 .

The achievement rates for complete MH for patients with baseline SF subscores of 0, 1, and 2 were 61.3\% (19/31), 35.6\% (21/59), and $24.1 \%$ (7/29), respectively, which demonstrated a significantly higher rate in patients with a lower baseline SF subscore in group B $(P=0.0050)$. Furthermore, the achievement rates for complete $\mathrm{MH}$ in patients with previous lesions reported as proctitis or "others" were $40.0 \%$ (20/50) and 39.1\% (27/69), respectively, which were similar in both lesion categories.

Table 3 shows the logistic regression analysis for CR at week 6. In the univariate analyses, we found that CR at week 6 was influenced by the following 8 variables: sex, body weight, clinical course (first attack or relapsing/remitting), duration of the present active phase, MMDAI, baseline RB subscore, baseline SF subscore, and physician's global assessment score. The multivariate analysis revealed that 3 variables-sex, clinical course (first attack or relapsing/remitting), and baseline SF subscorewere independently associated with CR at week 6 . The achievement rates for CR at week 6 for patients with a baseline SF subscore of 0 , 1 , or 2 were $61.3 \%$ (19/31), 39.0\% (23/59), and 37.9\% (11/29), respectively, which demonstrated a significantly higher rate in patients with a lower baseline $\mathrm{SF}$ subscore in group B $(P=0.0325)$. Finally, similar rates of CR achievement were observed in patients with or without proctitis: $46.0 \%(23 / 50)$ and $43.5 \%$ (30/69), respectively.

The explanatory variables of the univariate analysis were the same 23 items in the MH and CR analyses. However, there were differences in the variables found to be significantly associated with $\mathrm{MH}$ and $\mathrm{CR}$. Univariate analyses showed that the 4 most commonly related items included the duration of activity, MMDAI, RB subscore, and SF subscore. In the multivariate analysis after variable selection, only baseline SF subscore was significantly related to the explanatory variables for $\mathrm{MH}$ and CR, only MH was significantly related to the history of use of 5-ASA enema and suppositories and CR was significantly related to sex and clinical course (first attack or relapsing/remitting).

\section{Relationship between Complete MH or CR at Week 6 and RB Subscore or SF Subscore for 6 Weeks}

Table 4 shows the relationship between complete $\mathrm{MH}$ at week 6 and the RB or SF subscore at each visit. Table 4 shows the percentage of patients with complete MH at week 6 according to RB subscore at each visit. Stratified by RB subscore at week 2, 32 of 59 (54.2\%) patients with an RB subscore of 0,11 of 44 (25.0\%) patients with an RB subscore of 1, and 4 of 15 (26.7\%) patients with an RB subscore of 2 had MH at week 6. One patient with an RB subscore of 3 at week 2 had no MH at week 6 , resulting in a $0.0 \%(0 / 1)$ achievement rate. Patients with a lower RB subscore had a significantly higher rate of achieving $\mathrm{MH}$ than patients with a higher RB subscore (week 2: $P=0.0033$ ). Similarly, RB subscores at weeks 4 and 6 were significantly associated with the rate of complete MH at week 6 (week 4: $P=$ 0.0018, week 6: $P=0.0002$ ). Table 4 shows the percentage of patients with complete MH at week 6 according to SF subscore at each visit. Stratified by SF subscore at baseline, 19 of 31 (61.3\%) patients with an SF subscore of 0, 21 of 59 (35.6\%) patients with an SF subscore of 1, and 7 of 29 (24.1\%) patients with an SF subscore of 2 had MH at week 6. Patients with a lower SF subscore had a significantly higher rate of achieving MH than patients with a higher SF subscore (baseline: $P=0.0031$ ). Similarly, SF subscore at week 2 was significantly associated with the rate of complete $\mathrm{MH}$ at week $6(P=0.0360)$. However, SF subscore at week 4 was not significantly associated with the complete $\mathrm{MH}$ rate at week 6 .

Table 5 shows the results of the analysis evaluating the relationship between CR at week 6 and RB or SF subscore at each visit. Table 5 shows the percentage of patients achieving CR at week 6 according to RB subscore at each visit. Stratified by RB subscore at week 2, 38 of 59 (64.4\%) patients with an RB subscore of 0,13 of 44 (29.5\%) patients with an RB subscore of 1 , and 2 of 15 (13.3\%) patients with an RB subscore of 2 had CR at week 6 . The 1 patient with an RB subscore of 3 at week 2 
Table 4. Relationship Between Complete MH at Week 6 and Rectal Bleeding Subscores and Stool Frequency Subscores at Each Visit in BF (twice/day) Group

\begin{tabular}{|c|c|c|c|c|c|}
\hline & \multirow[b]{2}{*}{$\begin{array}{l}\text { Cate- } \\
\text { gory }\end{array}$} & \multirow[b]{2}{*}{ No. } & \multicolumn{2}{|c|}{ Complete $\mathrm{MH}$ at week 6} & \multirow[b]{2}{*}{$P$-value ${ }^{a}$} \\
\hline & & & $\begin{array}{c}\text { No. of } \\
\text { patients } \\
\text { with } \mathrm{MH}\end{array}$ & $\begin{array}{c}\text { Achievement } \\
\text { rate }(\%)\end{array}$ & \\
\hline \multicolumn{6}{|c|}{ Rectal bleeding subscore } \\
\hline \multirow[t]{4}{*}{ Baseline } & 0 & 0 & - & - & 0.0513 \\
\hline & 1 & 68 & 32 & 32/68 (47.1) & \\
\hline & 2 & 51 & 15 & $15 / 51(29.4)$ & \\
\hline & 3 & 0 & - & - & \\
\hline \multirow[t]{4}{*}{ Week 2} & 0 & 59 & 32 & $32 / 59(54.2)$ & $0.0033^{b}$ \\
\hline & 1 & 44 & 11 & $11 / 44(25.0)$ & \\
\hline & 2 & 15 & 4 & 4/15 (26.7) & \\
\hline & 3 & 1 & 0 & $0 / 1(0.0)$ & \\
\hline \multirow[t]{4}{*}{ Week 4} & 0 & 75 & 38 & $38 / 75$ (50.7) & $0.0018^{b}$ \\
\hline & 1 & 30 & 9 & 9/30 (30.0) & \\
\hline & 2 & 8 & 0 & $0 / 8(0.0)$ & \\
\hline & 3 & 0 & - & - & \\
\hline \multirow[t]{4}{*}{ Week 6} & 0 & 78 & 42 & 42/78 (53.8) & $0.0002^{c}$ \\
\hline & 1 & 29 & 5 & $5 / 29(17.2)$ & \\
\hline & 2 & 4 & 0 & 0/4 (0.0) & \\
\hline & 3 & 0 & - & - & \\
\hline \multicolumn{6}{|c|}{ Stool frequency subscore } \\
\hline \multirow[t]{4}{*}{ Baseline } & 0 & 31 & 19 & 19/31 (61.3) & $0.0031^{b}$ \\
\hline & 1 & 59 & 21 & $21 / 59(35.6)$ & \\
\hline & 2 & 29 & 7 & $7 / 29(24.1)$ & \\
\hline & 3 & 0 & - & - & \\
\hline \multirow[t]{4}{*}{ Week 2} & 0 & 56 & 28 & 28/56 (50.0) & $0.0360^{b}$ \\
\hline & 1 & 55 & 17 & 17/55 (30.9) & \\
\hline & 2 & 5 & 2 & $2 / 5(40.0)$ & \\
\hline & 3 & 2 & 0 & $0 / 2(0.0)$ & \\
\hline \multirow[t]{4}{*}{ Week 4} & 0 & 66 & 33 & 33/66 (50.0) & 0.0589 \\
\hline & 1 & 42 & 12 & 12/42 (28.6) & \\
\hline & 2 & 4 & 2 & $2 / 4(50.0)$ & \\
\hline & 3 & 1 & 0 & 0/1 (0.0) & \\
\hline \multirow[t]{4}{*}{ Week 6} & 0 & 69 & 33 & 33/69 (47.8) & 0.1170 \\
\hline & 1 & 40 & 13 & $13 / 40(32.5)$ & \\
\hline & 2 & 2 & 1 & 1/2 (50.0) & \\
\hline & 3 & 1 & 0 & 0/1 (0.0) & \\
\hline
\end{tabular}

${ }^{\mathrm{a}}$ Cochran-Armitage test.

${ }^{b} P<0.05$.

${ }^{\mathrm{c}} P<0.001$.

$\mathrm{MH}$, mucosal healing; $\mathrm{BF}$, budesonide 2-mg foam.
Table 5. Relationship Between CR at Week 6 and Rectal Bleeding Subscores and Stool Frequency Subscores at Each Visit in BF (twice) day) Group

\begin{tabular}{|c|c|c|c|c|c|}
\hline & \multirow[b]{2}{*}{$\begin{array}{l}\text { Cate- } \\
\text { gory }\end{array}$} & \multirow[b]{2}{*}{ No. } & \multicolumn{2}{|c|}{ CR at week 6} & \multirow[b]{2}{*}{$P$-value ${ }^{a}$} \\
\hline & & & $\begin{array}{l}\text { No. of } \\
\text { patients } \\
\text { with } \mathrm{MH}\end{array}$ & $\begin{array}{c}\text { Achievement } \\
\text { rate }(\%)\end{array}$ & \\
\hline \multicolumn{6}{|c|}{ Rectal bleeding subscore } \\
\hline \multirow[t]{4}{*}{ Baseline } & 0 & 0 & - & - & $0.0332^{b}$ \\
\hline & 1 & 68 & 36 & 36/68 (52.9) & \\
\hline & 2 & 51 & 17 & 17/51 (33.3) & \\
\hline & 3 & 0 & - & - & \\
\hline \multirow[t]{4}{*}{ Week 2} & 0 & 59 & 38 & $38 / 59(64.4))$ & $<0.0001^{d}$ \\
\hline & 1 & 44 & 13 & $13 / 44(29.5)$ & \\
\hline & 2 & 15 & 2 & 2/15 (13.3) & \\
\hline & 3 & 1 & 0 & 0/1 (0.0) & \\
\hline \multirow[t]{4}{*}{ Week 4} & 0 & 75 & 47 & $47 / 75(62.7)$ & $<0.0001^{\mathrm{d}}$ \\
\hline & 1 & 30 & 6 & $6 / 30(20.0)$ & \\
\hline & 2 & 8 & 0 & 0/8 (0.0) & \\
\hline & 3 & 0 & - & - & \\
\hline \multirow[t]{4}{*}{ Week 6} & 0 & 78 & 53 & 53/78 (67.9) & $<0.0001^{\mathrm{d}}$ \\
\hline & 1 & 29 & 0 & $0 / 29(0.0)$ & \\
\hline & 2 & 4 & 0 & 0/4 (0.0) & \\
\hline & 3 & 0 & - & - & \\
\hline
\end{tabular}

Stool frequency subscore

$\begin{array}{lrrrcr}\text { Baseline } & 0 & 31 & 19 & 19 / 31(61.3) & 0.0647 \\ & 1 & 59 & 23 & 23 / 59(39.0) & \\ & 2 & 29 & 11 & 11 / 29(37.9) & \\ \text { Week 2 } & 0 & 56 & 36 & 36 / 56(64.3) & 0.0003^{\mathrm{c}} \\ & 1 & 55 & 15 & 15 / 55(27.3) & \\ & 2 & 5 & 2 & 2 / 5(40.0) & \\ & 3 & 2 & 0 & 0 / 2(0.0) & \\ \text { Week 4 } & 0 & 66 & 43 & 43 / 66(65.2) & <0.0001^{d} \\ & 1 & 42 & 10 & 10 / 42(23.8) & \\ & 2 & 4 & 0 & 0 / 4(0.0) & \\ & 3 & 1 & 0 & 0 / 1(0.0) & \\ \text { Week 6 } & 0 & 69 & 49 & 49 / 69(71.0) & <0.0001^{d} \\ & 1 & 40 & 4 & 4 / 40(10.0) & \\ & 2 & 2 & 0 & 0 / 2(0.0) & \\ & 3 & 1 & 0 & 0 / 1(0.0) & \end{array}$

${ }^{\mathrm{a}}$ Cochran-Armitage test.

${ }^{b} P<0.05$.

${ }^{\mathrm{c}} P<0.001$.

${ }^{\mathrm{d}} P<0.0001$.

$\mathrm{CR}$, clinical remission; $\mathrm{BF}$, budesonide 2-mg foam; $\mathrm{MH}$, mucosal healing. 
had no CR at week 6 (achievement rate of $0.0 \%$ ). Patients with smaller $\mathrm{RB}$ subscores tended to be more likely to achieve $\mathrm{CR}$ $(P<0.0001)$. RB subscores at both week 4 and 6 were significantly associated with the rate of achievement of $\mathrm{CR}$ at week 6 (week 4: $P<0.0001$, week 6: $P<0.0001$ ).

Table 5 shows the percentage of patients with CR at week 6 according to SF subscore at each visit. SF subscores at week 2, week 4 , and week 6 were significantly associated with the induction rate of CR at week 6 ( $P=0.0003, P<0.0001, P<0.0001$, respectively). Both $\mathrm{RB}$ and $\mathrm{SF}$ subscores during treatment were shown to be predictors of CR at week 6 .

\section{Safety}

Adverse events from treatment in the 2 clinical studies have already been reported. ${ }^{12,13}$ Briefly, the most common were steroid-related abnormal laboratory test values: a $48.3 \%$ decrease in plasma cortisol and a $35.0 \%$ decrease in plasma corticotrophin in group B. There were no other steroid-related adverse events identified in this pooled analysis.

\section{DISCUSSION}

This was a post hoc analysis of pooled data from phase II and III clinical trials evaluating the efficacy and safety of BF treatment in UC patients. We conducted this analysis to identify the demographic and clinical factors affecting prognosis and analyzed the relationship between complete $\mathrm{MH}$ or $\mathrm{CR}$ and $\mathrm{RB}$ or SF subscores from baseline to week 6 to identify predictors of the therapeutic effect of BF. In this analysis, improvement of clinical symptoms (i.e., improvement in the RB or SF subscores) was clearly observed after 5 days (RB subscore) and after 2 days (SF subscore) of BF treatment. Based on this finding, it was suggested that topical BF might contribute to early symptom relief.

As a result of the multivariate analysis to identify the background patient characteristics that may affect treatment outcomes (efficacy endpoints), we found that complete MH was influenced by the baseline SF subscore and the use of a 5-ASA enema or suppository. Approximately $45 \%$ of patients had previously received a 5-ASA rectal enema or suppository. The multivariate analysis showed a statistically significant difference between patients previously treated with local 5-ASA treatment and those not previously treated. It has been reported that local 5-ASA treatment results in $\mathrm{MH}^{16}$ and patients who had a history of local 5-ASA treatment and were enrolled in the original clinical study were likely to have an insufficient $\mathrm{MH}$ re- sponse. Thus, it is possible that they had more severe disease characteristics than patients without a history of local 5-ASA treatment. In order to eliminate the effect of 5-ASA topical product use, specific exclusion criteria (i.e., patients using mesalazine preparations [enema or suppository] or salazosulfapyridine preparation [suppository] 1-week before the date of qualification were excluded from the study) were set at the time of the clinical trial to ensure a l-week washout period. Therefore, the efficacy of BF was considered to have been adequately evaluated.

In the multivariate analysis, CR was influenced by sex, clinical course (first attack or relapsing/remitting), and baseline SF subscore. Male patients were more likely to achieve CR at week 6 than female patients. Other studies have shown differences in efficacy by sex, with women more likely to respond than men ${ }^{9}$ and a higher relapse rate in women than in men. $^{17}$ The results of this analysis suggest that further investigations into sex differences are warranted.

Additionally, relapsing/remitting patients were more likely to achieve CR at week 6 than those with a first attack. In this analysis, however, 105 patients had relapsing/remitting disease while only 13 were having their first attack. Thus, it is difficult to draw any conclusions from these data. We presume that the moderate symptoms of patients with relapsing/remitting UC could be more easily controlled by the treatment and patients with refractory relapsing/remitting UC were not registered in this study. However, some patients with a first attack could have perceived their symptoms as mild to moderate but may have actually had more severe disease and, thus, it may have been more difficult for them to achieve CR by week 6 . Additionally, both RB and SF subscores during treatment seemed to be predictive of achievement of CR at week 6 . As we only examined a small number of cases, further research with larger sample sizes must be conducted to clarify this result.

A low baseline SF subscore before BF treatment was found to be a characteristic of patients who achieved complete $\mathrm{MH}$. We consider that the retention time of the compound solution within the inflammatory lesion may be attributable to this effect. Further, our analyses suggest that a normal SF at the initiation of treatment might be the most relevant patient characteristic for choosing $\mathrm{BF}$ treatment because complete $\mathrm{MH}$ can be achieved under this condition. According to the study by Brunner et al., ${ }^{3}$ it takes 6 hours to reach the maximum drug distribution of BF. Thus, patients who had a high SF subscore and frequent defecations may not have retained the drug for a sufficient amount of time, which may have resulted in a de- 
creased absorption volume of BF and a weakened treatment effect. This finding highlights the need to follow the recommendations on the timing of $\mathrm{BF}$ administration in relation to the patient's bowel movement patterns, such as avoiding BF treatment immediately after eating when peristaltic movements tend to increase. In this post hoc analysis, the achievement rates of complete $\mathrm{MH}$ or CR among patients with proctitis and other types of previous lesions were similar. These results are consistent with previously reported pharmacokinetic data ${ }^{3}$ from a study conducted in Austria that evaluated the diffusion of a single rectal administration of BF labeled with ${ }^{99 \mathrm{~m}} \mathrm{Tc}$. That study reported that $\mathrm{BF}$ reached the sigmoid colon in all 12 patients with mild-to-moderately active UC.

A recent study ${ }^{9}$ indicated that incomplete $\mathrm{MH}$ was a factor related to a nonbeneficial outcome (use of immunosuppressive agents, hospitalization, and colectomy) in patients who were treated with steroids. The BF dose of $2 \mathrm{mg} / 25 \mathrm{~mL}$ administered twice a day was approved because the achievement rate for complete $\mathrm{MH}$ was higher in the group receiving $\mathrm{BF}$ twice a day than in the group receiving $\mathrm{BF}$ once a day in the phase II study. ${ }^{18}$ Although topical treatment is effective for UC patients, it is often used only on demand because of patient unacceptance or inconvenience. ${ }^{14}$

We found that RB subscores after week 2 during BF treatment were associated with the achievement of complete $\mathrm{MH}$ at week 6 in this analysis. However, there was no significant trend at weeks 4 and 6, suggesting that SF subscore during treatment was less predictive of achieving complete $\mathrm{MH}$ at week 6 than RB subscore. Thus, SF subscores after week 4 during BF treatment did not necessarily correlate with achievement of complete MH at week 6 . The SF subscores up to week 2 could be useful predictors of complete $\mathrm{MH}$ at week 6 , but not from week 4 onwards. These results suggest that RB subscore after week 2 may be useful as a predictive factor of complete $\mathrm{MH}$ achievement at week 6 . In some patients, RB did not resolve early in treatment, but $\mathrm{MH}$ was achieved at week 6 . Therefore, it is also important to observe and examine the status of $\mathrm{RB}$ in each patient in order to decide whether to continue treatment. Conversely, the complete $\mathrm{MH}$ rate at week 6 was $30 \%$ (9/30) with an RB subscore of 1 at week 4 , but $0 \%(0 / 8)$ with an RB subscore of 2 at week 4 . This suggests the need to decide whether to continue treatment after week 4 for UC patients with an RB subscore of 2 or higher. However, because the number of patients in this analysis was small, it seemed important to decide upon treatment continuation by evaluating the symptoms of patients individually.
We conclude that it is important to monitor RB after initiation of $\mathrm{BF}$ treatment to achieve complete $\mathrm{MH}$ at week 6 , and it is recommended to determine the adequacy of treatment continuation based on the RB subscores at weeks 2 and 4 .

Aside from evaluating the SF subscore at baseline, RB subscore after week 2 is significantly associated with complete $\mathrm{MH}$ and thus may help predict the effect of BF treatment. Regarding the adherence to treatment, we consider that this could be improved once patients receive the appropriate instructions for administering the medication, and patient awareness is increased regarding the effects of continuing treatment twice daily up to week 6 .

As this pooled analysis did not yield any notable safety findings, it is worth iterating the results of the 2 individual trials. ${ }^{12,13}$ In one study, ${ }^{12}$ the incidence of adverse effects in the placebo group was lower (29.6\%) than that in the BF bid group (67.9\%), while in the other study, ${ }^{13}$ the incidence was similar in the placebo (40.3\%) and BF (45.3\%) groups. No serious treatment-related adverse effects or deaths were reported in either study. In both studies, ${ }^{12,13}$ glucocorticoid-related adverse events occurring only in the BF groups were decreased plasma concentrations of cortisol and adrenocorticotropin; however, the levels returned to normal after the treatment was terminated.

This analysis has some limitations, including the relatively low number of patients. Additionally, this was a post hoc analysis of pooled data from 2 existing clinical trials, and multiplicity was not considered. Additional studies are needed to assess the possibility of another relationship between $\mathrm{MH}$ or $\mathrm{CR}$ and changes in the baseline RB or SF subscores in clinical practice.

In conclusion, our analysis suggests 3 patient characteristics, namely, history of 5-ASA topical product use, normal SF at baseline, and elimination of $\mathrm{RB}$ after week 2 were found to be associated with complete $\mathrm{MH}$ at week 6 with twice-daily $\mathrm{BF}$ treatment. These characteristics could be useful predictors of BF treatment effect.

\section{FINANCIAL SUPPORT}

This work was supported by EA Pharma Co., Ltd. and Kissei Pharmaceutical Co., Ltd.

\section{CONFLICT OF INTEREST}

Hibi T is an editor of Intestinal Research, has received grants, personal fees, and/or other funds from Zeria Pharmaceutical 
Co., Ltd., EA Pharma Co., Ltd., Nippon Kayaku Co., Ltd., Mochida Pharmaceutical Co., Ltd., Kissei Pharmaceutical Co., Ltd., Takeda Pharmaceutical Co., Ltd., Mitsubishi Tanabe Pharma Corporation, Janssen Pharmaceutical K.K., Pfizer Japan Inc., AbbVie GK, JIMRO Co., Ltd., and Otsuka Holdings Co., Ltd.

Naganuma $\mathrm{M}$ has received grants, personal fees, and/or nonfinancial support from EA Pharma Co., Ltd., Mochida Pharmaceutical Co., Ltd., Kissei Pharmaceutical Co., Ltd., Mitsubishi Tanabe Pharma Corporation, Takeda Pharmaceutical Co., Ltd., JIMRO Co., Ltd., Kyorin Pharmaceutical Co., Ltd., Janssen Pharmaceutical K.K., Pfizer Japan Inc., AbbVie GK, and Thermo Fisher Scientific Inc.

Oda E has received grants from EA Pharma Co., Ltd. and Kissei Pharmaceutical Co., Ltd.

Yamada Y and Chujoh Y are employees of EA Pharma Co., Ltd. Yoshihara R is an employee of Kissei Pharmaceutical Co., Ltd.

Watanabe $\mathrm{M}$ has received grants, personal fees and/or other funds from Takeda Pharmaceutical Co., Ltd., Eisai Co., Ltd., Astellas Pharma Inc., Nippon Kayaku Co., Ltd., Mochida Pharmaceutical Co., Ltd., Pfizer Japan Inc., Kissei Pharmaceutical Co., Ltd., Mitsubishi Tanabe Pharma Corporation, Zeria Pharmaceutical Co., Ltd., EA Pharma Co., Ltd., Kaken Pharmaceutical Co., Ltd., Alfresa Pharma Corporation, Daiichi Sankyo Co., Ltd., Taiho Pharmaceutical Co., Ltd., Ayumi Pharmaceutical Corporation, Miyarisan Pharmaceutical Co., Ltd., MSD K.K., Janssen Pharmaceutical K.K., Celltrion Healthcare Co., Ltd., Celgene K.K., Eli Lilly Japan K.K., JIMRO Co., Ltd., Gilead Sciences, Inc., Asahi Kasei Medical Co., Ltd., Kyorin Pharmaceutical Co., Ltd., AbbVie GK, Kyowa Hakko Kirin Co., Ltd., Toray Industries, Inc., Chugai Pharmaceutical Co., Ltd., and Fujirebio Inc. No other potential conflict of interest relevant to this article was reported.

\section{AUTHOR CONTRIBUTION}

Conceptualization: Hibi T, Oda E, Yamada Y, Chujoh Y, Yoshihara R. Methodology: Hibi T, Oda E, Yamada Y, Chujoh Y, Yoshihara R. Formal analysis: Oda E. Investigation: Hibi T, Naganuma M, Oda E, Yamada Y, Chujoh Y, Yoshihara R, Watanabe M. Writing - original draft: Hibi T, Naganuma M, Oda E, Chujoh Y, Yoshihara R. Writing - review and editing: all authors. Approval of final manuscript: all authors.

\section{ORCID}

Hibi T

Naganuma M https://orcid.org/0000-0002-6256-1204

https://orcid.org/0000-0002-4244-3243
Oda E

Yamada Y

Chujoh Y

Yoshihara R

Watanabe M https://orcid.org/0000-0001-8183-3773

https://orcid.org/0000-0002-7361-0956

https://orcid.org/0000-0002-7315-1040

https://orcid.org/0000-0002-5475-9544 https://orcid.org/0000-0003-1063-5213

\section{ACKNOWLEDGEMENTS}

The authors would like to thank the patients, investigators, and study teams who were involved in the phase II and III clinical trials for budesonide 2-mg foam. This study was supported by EA Pharma Co., Ltd. and Kissei Pharmaceuticals Co., Ltd. The authors received medical writing assistance from Mr. Noritsugu Hirasawa (AC Medical Inc., Tokyo, Japan) for preparation of the initial and final drafts of the manuscript and editorial support from Dr. Keyra Martinez Dunn (Edanz Medical Writing, Fukuoka, Japan), which were funded by EA Pharma Co., Ltd. and Kissei Pharmaceutical Co., Ltd.

\section{REFERENCES}

1. Harbord M, Eliakim R, Bettenworth D, et al. Third European evidence-based consensus on diagnosis and management of ulcerative colitis. part 2: current management. J Crohns Colitis 2017;11:769-784.

2. Ko CW, Singh S, Feuerstein JD, et al. AGA clinical practice guidelines on the management of mild-to-moderate ulcerative colitis. Gastroenterology 2019;156:748-764.

3. Brunner M, Vogelsang H, Greinwald R, et al. Colonic spread and serum pharmacokinetics of budesonide foam in patients with mildly to moderately active ulcerative colitis. Aliment Pharmacol Ther 2005;22:463-470.

4. Hochhaus G, Derendorf H, Möllmann HW, Barth J, Hochhaus R. Pharmacodynamic aspects of glucocorticoid action. In: Mollmann HW, May B, eds. Glucocorticoid therapy in chronic inflammatory bowel disease: from basic principles to rational therapy. Dordrecht: Kluwer, 1993:61-79.

5. Brattsand R, Thalén A, Roempke K, Källström L, Gruvstad E. Influence of 16 alpha, 17 alpha-acetal substitution and steroid nucleus fluorination on the topical to systemic activity ratio of glucocorticoids. J Steroid Biochem 1982;16:779-786.

6. Gross V, Bar-Meir S, Lavy A, et al. Budesonide foam versus budesonide enema in active ulcerative proctitis and proctosigmoiditis. Aliment Pharmacol Ther 2006;23:303-312.

7. Suzuki Y, Iida M, Ito H, et al. $2.4 \mathrm{~g}$ mesalamine (Asacol $400 \mathrm{mg}$ tablet) once daily is as effective as three times daily in mainte- 
nance of remission in ulcerative colitis: a randomized, noninferiority, multi-center trial. Inflamm Bowel Dis 2017;23:822832.

8. Yoshino T, Yamakawa K, Nishimura S, Watanabe K, Yazumi S. The predictive variable regarding relapse in patients with ulcerative colitis after achieving endoscopic mucosal healing. Intest Res 2016;14:37-42.

9. Ardizzone S, Cassinotti A, Duca P, et al. Mucosal healing predicts late outcomes after the first course of corticosteroids for newly diagnosed ulcerative colitis. Clin Gastroenterol Hepatol 2011;9:483-489.

10. Frøslie KF, Jahnsen J, Moum BA, Vatn MH; IBSEN Group. Mucosal healing in inflammatory bowel disease: results from a Norwegian population-based cohort. Gastroenterology 2007; 133:412-422.

11. Yokoyama K, Kobayashi K, Mukae M, Sada M, Koizumi W. Clinical study of the relation between mucosal healing and long-term outcomes in ulcerative colitis. Gastroenterol Res Pract 2013;2013:192794.

12. Naganuma M, Aoyama N, Suzuki Y, et al. Twice-daily budesonide 2-mg foam induces complete mucosal healing in patients with distal ulcerative colitis. J Crohns Colitis 2016;10:828-836.

13. Naganuma M, Aoyama N, Tada T, et al. Complete mucosal healing of distal lesions induced by twice-daily budesonide 2-mg foam promoted clinical remission of mild-to-moderate ulcerative colitis with distal active inflammation: double-blind, randomized study. J Gastroenterol 2018;53:494-506.

14. Matsuoka K, Kobayashi T, Ueno F, et al. Evidence-based clinical practice guidelines for inflammatory bowel disease. J Gastroenterol 2018;53:305-353.

15. Sandborn WJ, Kamm MA, Lichtenstein GR, Lyne A, Butler T, Joseph RE. MMX Multi Matrix System mesalazine for the induction of remission in patients with mild-to-moderate ulcerative colitis: a combined analysis of two randomized, doubleblind, placebo-controlled trials. Aliment Pharmacol Ther 2007; 26:205-215.

16. Kobayashi K, Hirai F, Naganuma M, et al. A randomized clinical trial of mesalazine suppository: the usefulness and problems of central review of evaluations of colonic mucosal findings. J Crohns Colitis 2014;8:1444-1453.

17. Höie O, Wolters F, Riis L, et al. Ulcerative colitis: patient characteristics may predict 10-yr disease recurrence in a European-wide population-based cohort. Am J Gastroenterol 2007; 102:1692-1701.

18. Package insert of budesonide 2-mg foam. https://pins.japic. or.jp/pdf/newPINS/00067157.pdf. Accessed 30 August, 2019. 